approaches. For von Weizäcker it was Einstein's "aloofness" not his greater political understanding that permitted Einstein to act politically while others, as von Weizsäcker puts it, "conceded apparently or truly - to believe in the political prejudices of the time in order to function concretely in their midst".

Similarly Banesh Hoffman presents only a superficial view of Einstein's Zionism by failing to situate Einstein's support for the state of Israel in the context of the intense anti-semitism of Europe between the Wars. As Einstein said in 1938:

"Rarely since the conquest of Jerusalem by Titus has the Jewish community experienced a period of greater oppression than prevails at the present time".

Einstein supported the Zionist cause pragmatically and I think it is a mistake to present him as a narrow nationalist. This was his appraisal of the situation in 1930:

"I saw how schools, comic papers and innumerable other forces of the Gentile majority undermined the confidence of even the best of my fellow-Jews and felt that this could not be allowed to continue. Then I realised that only a common enterprise dear to the heart of Jews all over the world could restore this people to health'".

But in spite of the fact that the collection does not live up to the promise of its title there is still much to interest the casual reader. Dennis Sciama offers a nice concise review of the current position in cosmology and Roger Penrose delivers an informative and very nearly comprehensible account of black holes. And of continuing interest are the personal reminiscences of Einstein. Walther Gerlach on Einstein and quantum theory and John Wheeler on Einstein's Princeton days are new additions to the Einstein archive. Particularly welcome is the inclusion of Einstein's last lecture which along with the transcript of his answers to the questions give a vivid picture of Einstein in action as a thinker. Finally, Wolfgang Yourgrau's portrait, "Einstein - and the Vanity of Academia" is a fresh and irreverant view of the German academy in the 1920s. Yourgrau may have gone too far in reporting his mother's shocked observation that Einstein had dandruff, but I think Einstein would approve. The idea of a god with dandruff would have amused him immensely.

Joe Schwartz

Joe Schwartz is on leave from the City University of New York.

\section{Communicative abilities}

Studies of Language Thought and Verbal Rommetveit and R. Blakar. Pp. 466. Communication. Edited by R. (Academic: London and New York, 1979.) $£ 22.80$.

OVER the past 20 years Rommetveit has been developing a particular approach to the psychology of language, thought and communication. He calls his position a "social-cognitive" one and it contrasts in particular with the psycholinguisticexperimental approach with its focus on single sentences. Rommetveit has always held that such an approach has led to gross simplifications which could never be of use in comprehending the complexities of the mechanisms underlying our communicative abilities.

This book is a collection of articles by Rommetveit and his collaborators in Oslo. It is a mixture of theoretical statements and

experimental articles. The 35 articles are of very mixed value and there is little attempt to link together the varied work which ranges from word recognition to the verbal maintenance of sex rules in Norwegian. The experimental section of 27 articles would have benefited from severe pruning.

There is better value in the theoretical articles (most of which are not easily available in their original places) where Rommetveit draws on a number of linguistic and philosophical traditions in discussing the nature of language and communication and moving over into hermeneutics and social psychology. I didn't always succeed in understanding, and quite frequently disagreed but found myself stimulated agreeably often. However, Rommetveit never achieves the synthesis he aims for.

John Morton

John Morton is a member of the scientific staff of the MRC Applied Psychology Unit, Cambridge, UK.

\section{Vibrations of molecular crystals}

Molecular Vibrations in Crystals. By J.C. Decius and R.M. Hexter. Pp. 391 (McGraw-Hill: New York and London, 1979.) £15.60; $\$ 29.50$

MANY textbooks discuss in considerable detail the lattice vibrations of simple covalent and ionic crystals but this book is unique in dealing with the vibrations of molecular crystals. As these are of the type that most chemical solid-state spectroscopists deal with, this book will be of interest and value to the growing number of scientists who study experimentally the vibrations of molecular crystals using infrared, Raman or neutron spectroscopy or to theoreticians carrying out lattice dynamical calculations on molecular crystals. It is jointly written by two authors with an international reputation for their contributions to the vibrational spectra of molecular crystals using infrared spectroscopy. In many ways the book is a natural extension of the now classic work on Molecular Vibrations by Wilson, Decius and Cross (McGraw-Hill) which deals with isolated molecules.

The original approach used in this book is to emphasise a representation based on internal coordinates and thus to take advantage of molecular integrity where it is appropriate, but to permit its modification by perturbation theory. In the internal coordinate representation the dynamical matrix becomes the familiar FG matrix of Wilson, Decius and Cross. A new method of calculation of this matrix, valid throughout the Brillouin zone is developed in this treatise.

Many chemical spectroscopists are not familiar with the splitting of longitudinal and transverse optical modes; they will therefore find useful the review of this important topic which is treated both from the traditional macro-scopic point of view as well as with microscopic theory. This has not previously been so carefully done.

Six different molecular crystals $(\mathrm{HCN}$, $\mathrm{CO}_{2}, \mathrm{CHI}_{3}, \mathrm{NaN}_{3}$ and $\mathrm{NaClO}_{3}$ ) are used as detailed examples to consider infrared and Raman spectra and their selection rules. In all cases, spectra are discussed with respect to crystal splittings and polarisations. The current theories of these splittings are carefully examined, compared and evaluated in molecular as well as in ionic crystals. The expression of intermolecular coupling of vibration in both excitation and phonon coordinates is contrasted, and the basis and extent of their equivalence is discussed.

The theory of multiphonon spectra is thoroughly reviewed, covering selection rules, intensity distribution and origin in crystal anharmonicity. The Van Hove and Phillips theories of critical points are carefully explained. In one example, Iodoform-calculated phonon dispersion curves are presented and their consequences are compared with experiment.

In the final chapter, the spectra of impure crystals, both ionic and molecular, are discussed, together with a simple introduction to Green's function treatment of isotopically impure crystals.

The authors are to be congratulated on producing a well written definitive work that will appeal to physicists as well as chemists interested in molecular crystals. This book is likely to prove useful as a text for postgraduate courses on infrared and Raman spectra of solids, self-study reading for students starting research in the subject and as a guide to the extensive literature in this field.

G.R. Wilkinson

G.R. Wilkinson is Professor of Physics at King 's College, University of London, UK. 